LÁSZLÓ HORVÁTH

\title{
EINE VERGESSENE ÜBERSETZUNG DES JANUS PANNONIUS
}

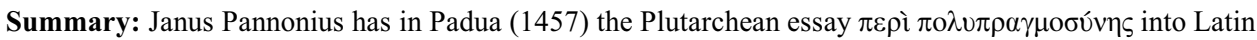
translated. In order to advocate his choice on the Latin equivalent of the Greek compound in the title (i.e. negotiositas - Gellius' later rejected proposal) Janus proposes at length his linguistic and philological arguments in the introductory letter to the Latin text. On the basis of a detailed analysis of Janus' references the author of the article demonstrates that Janus possessed a collection of ancient "referencebooks", since in his quotations he rather drew on Nonius Marcellus, Gellius and Quintilianus, instead of Cicero's text. Apart from Gellius' name Janus fails to mention the other two, although one of his Ciceroquotations occurs only in Nonius Marcellus' work De compendiosa doctrina and it is fallen out of the Ciceronian manuscript-tradition. So we can define a further group of books (codices) which Janus later so bitterly missed in Hungary. Janus Pannonius' proposal (De negotiositate) was finally replaced (if ever consulted) by Erasmus' De curiositate.

Key words: Janus Pannonius, negotiositas, Nonius Marcellus, Gellius, Quintilianus, Cicero, De curiositate, Plutarch.

Die Sekundärliteratur über die griechisch-lateinische Übersetzungstechnik des Janus Pannonius hat schon weitgehende Ergebnisse erbracht. Diese Studien, wie die Artikel von Zsigmond Ritoók, sind hauptsächlich einer direkten Analyse der lateinischen Texte gewidmet. ${ }^{1} \mathrm{Zu}$ diesen direkten erkannten philologischen Ergebnissen möchte ich in diesem Aufsatz einige Beobachtungen über die Umstände und die weitgehende Gründlichkeit der Übersetzungen hinzufügen, welche ich indirekt, nämlich aus einem Brief des Janus Pannonius gewonnen zu haben meine. Die erste Anregung zu diesem Thema hatte ich vor vielen Jahren von Prof. Zsigmond Ritoók bekommen. Vor zwei Jahren hat mich der Artikel von Prof. István Kapitánffy über das Vocabularium des Janus inspiriert das Thema wieder aufzugreifen. ${ }^{2}$ Er hat den ersten Aufsatz gelesen und kommentiert, aber wir konnten über seine Anmerkungen und Hinweise nicht mehr diskutieren. Leider verspätet, aber mit Dankbarkeit widme

${ }^{1}$ JuHÁsz László, De Iano Pannonio interprete Graecorum (Szeged, 1928), RitoóK Zsigmond, „Janus Pannonius görög versfordításai“ (Griechische Gedichtübersetzungen von Janus Pannonius). In: Janus Pannonius (Tanulmányok) KARdOS Tibor, V. KovÁcs Sándor (szerk.) (Budapest, 1975) (im weiteren $J P T$ ) 407-38.

${ }^{2}$ KAPITÁNFFY István, „Aristophanes, Triklinios, Guarino und Janus Pannonius“ Act.Ant.Hung. 36 (1995) 351-7. 
ich diesen Aufsatz seinem Andenken, in Erinnerung an meinen hochverehrten Professor und älteren Kollegen.

Janus Pannonius hat während seiner Studienjahre in Italien unter anderem $\pi \varepsilon \rho \grave{~}$ $\pi \circ \lambda \cup \pi \rho \alpha \gamma \mu \circ \sigma u ́ v \eta \varsigma$ „Über die Neugierde“ von Plutarch ins Lateinische übersetzt. ${ }^{3} \mathrm{Zu}$ dieser Übersetzung hat der Poet seine längste Einführung, einen an seinen Freund Marco Aurelio adressierten Brief geschrieben. (Der Text dieses Briefes befindet sich im Anhang.)

Der Inhalt dieses Briefes ist nur der potentiellen Übersetzung eines einzigen Wortes, nämlich des Titelwortes, gewidmet. In seinem Bestreben, eine lateinische Parallele des griechischen zusammengesetzten Wortes „, $\pi \lambda \nu \pi \rho \alpha \gamma \mu о \sigma 0 ́ v \eta “ z u$ finden, dienen Janus Pannonius die Texte und Argumente der römischen Autoren als Ausgangspunkt. In erster Linie sind es die Äußerungen des Gellius, die er benutzt und kritisiert (Gell.Noct.Att. XI.16. und IV.9). Obwohl Janus Pannonius sicherlich nicht etwas Neues in Betracht gezogen hat, also Unautorisiertes als eine sprachliche Neuerung einführen wollte, zeigen ihn seine Denkweise und Formulierung sowie seine stilistischen Bemerkungen als einen durchaus geeigneten Diskussionspartner des Gellius. Teile:

Nach der kurzen Widmung gliedert sich die briefliche Abhandlung in zwei

I. Zeilen 11-22. Janus stellt die Meinung des Gellius vor, daß er den antiken Autor (Gellius) anscheinend mit der Äußerung des von Gellius hochgeschätzten Nigidius Figulus in Frage stellt (letztere: Z. 19-22). ${ }^{4}$ Das heißt, die Anwendung von negotiositas ist völlig passend, weil solche Wortbildungungen nach Meinung des $\mathrm{Ni}$ gidius Figulus immer rein negative Bedeutung haben. Diese Meinung des Nigidius, mit der Janus Pannonius die Behutsamkeit des Gellius abweist, kann man ebenfalls im Werk des Gellius lesen, allerdings in einem anderen Kapitel, wo es um allgemeine Fragen der lateinischen Wortbildung geht. ${ }^{5}$

Doch - und darüber schweigt Janus Pannonius - Gellius präzisiert in dem gerade erwähnten Kapitel seines Werkes die Meinung des Nigidius. Er sagt, daß die auf solche Weise gebildeten Adjektive zweierlei Bedeutungen haben können. Es hänge davon ab, was für eine Bedeutung die ursprünglichen Begriffe hatten, aus denen die Neuerungen gebildet worden waren. Demgemäß können sie entweder eine positive oder eine negative Eigenschaft ausdrücken. ${ }^{6}$ Warum es so ist, daß es manchmal

\footnotetext{
${ }^{3}$ Plut.Mor. 515.B-523.B. Die übliche Übersetzung des Titels lautet: De curisositate.

${ }^{4}$ Gell.Noct.Att. XI.16.

${ }^{5}$ Meiner Meinung nach können wir das Werk des Nigidius Figulus aus dem virtuellen Katalog der Janus-Bibliothek gewiß streichen. Nigidius sollte nicht einmal zu denjenigen Büchern gezählt werden, aus denen einige Zitate oder Referenzen von Janus stammen können. Sehr vorsichtig, aber das Gegenteil behauptet Csaba CSAPODI, „Janus Pannonius könyvei és pécsi könyvtára“ in: $J P T$ 199, daß nämlich ein Nigidius-Kodex vielleicht verloren gegangen sein könnte.

${ }^{6}$ In seinem Brief sagt Janus expressis verbis nicht, daß in der Meinung des Nigidius allerlei Maßlosigkeit, bestimmt aber etwas Negatives war. Wir können es aber für sicher halten, daß Janus auf diese Weise die Meinung des Nigidius für sich interpretiert hatte, weil es einerseits aus seiner Argumentationsweise klar hervorgeht und sich andererseits Gellius klar darüber ausspricht:

Quod si, ut ait Nigidius, omnia istiusmodi inclinamenta nimium ac praeter modum significant et idcirco in culpas cadunt ... (Gell.Noct.Att. IV.9.12).
}

Acta Ant. Hung. 41, 2001 
gleich gebildete Adjektive gibt, die eine positive Bedeutung haben, das ist die Frage des Gellius: ... an propterea, quia illis quidem, quae supra posui (sc. vinosus, mulierosus etc.) adhibendus est modus quidam necessarius? Nam et gratia, si nimia atque inmodica, et mores, si multi atque varii, et verba, si perpetua atque infinita et obtundentia, et fama, si magna et inquieta et invidiosa sit, neque laudabilia neque utilia sunt; ingenium autem et officium et forma et disciplina et consilium et victoria et facundia sicut ipsae virtutum amplitudines nullis finibus cohibentur, sed quanto maiora auctioraque sunt, multo etiam tanto laudatiora sunt. ${ }^{7}$

Da der Begriff negotium und somit die Bildung negotium - negotiosus eher zu der letzteren also der positiven Gruppe gehört - wenn wir nur daran denken, daß das Adjektiv negotiosus in der lateinischen Literatur in der Bedeutung, geschäftig, tätig ‘ vorkommt und das unbegrenzte Betreiben des negotium - ,Tätigkeit' keine negative Erscheinung ist -, ist es völlig greifbar, warum Gellius die Verwendung von negotiositas als Übersetzung eines negativen griechischen Begriffs letztlich verworfen hatte.

Man kann sich leicht vorstellen, daß Gellius eine ähnliche Meinung über die Wortbildung cura - curiosus hatte, um so mehr, weil sich die römischen Autoren nicht darüber einig waren, daß curiosus etwas Negatives bedeutete. Für Gellius könnte cura positiv sein. Darauf reflektiert Janus Pannonius mit dem schweigenden Hinweis in Z. 15-16. Nur wenn wir annehmen, daß Janus die Argumente der beiden antiken Autoren analysiert hatte, wird es - meiner Meinung nach - völlig verständlich, warum Janus in seiner Argumentation unerwartet erwähnt, daß Gellius sehr oft curiosus in der Bedeutung accuratus , sorgfältig' benutzt. ${ }^{8}$ Man könnte auch nicht erwarten, daß Gellius die curiositas als eine mögliche Übersetzung der $\pi \circ \lambda v \pi \rho \alpha \gamma \mu \mathrm{o}-$

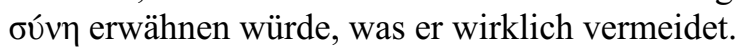

In der Ablehnung der negotiositas spielt für Gellius die ungewöhnliche Lautung des Wortes ebenfalls eine bedeutende Rolle. ${ }^{9}$ Darauf reflektiert auch Janus ein wenig später, wie er es immer mit allen Bemerkungen des Gellius tut. Dieses Letztere spricht auch für das intensive Bestreben des Janus Pannonius, der die Einwände des Gellius von Punkt zu Punkt widerlegen möchte. Wie es wir sehen konnten, stammen die ersten Gegenbeweise, obwohl ein wenig einseitig, aber sehr wohlklingend, von Gellius selbst.

II. Zeilen 22-36. Im zweiten Teil seines Briefes versucht Janus, die von Gellius verworfene Version mit weiteren Testimonien zu unterstützen. Beispiele aus Cicero und Catull folgen als Beweise, daß einerseits solche Wortbildungen in malum tantum interpretiert werden können, also nur eine negative Bedeutung haben können (Z. 28) und andereseits keine Gefahr der Ungewöhnlichkeit besteht.

Insgesamt sind sechs loci angeführt. Eine ansehnliche Zahl, besonders wenn Janus Pannonius alle aus dem Gedächtnis angeben mußte. Es gibt beinahe wörtliche

${ }^{7}$ Gell.Noct.Att. IV.9,12-14.

${ }^{8}$ Dieser kaum eingeleitete Hinweis könnte sicherlich die weitgehende Aufmerksamkeit des Janus preisen, aber es könnte auch unseren Verdacht bestätigen: Janus hat hier nämlich wieder auf die Nigidius korrigierenden Bemerkungen des Gellius (IV.9) reflektiert und demnach die Schlußfolgerung gezogen: Der Gebrauch des accuratus in der Bedeutung von curiosus ist bei Gellius bewußt und konsequent.

${ }^{9}$ Gell.Noct.Att. XI.16.4. 
Übereinstimmungen auch mit Gellius. Wenn wir aber die Testimonien und Zitate nacheinander prüfen, wird klar, daß sich Janus Pannonius diesmal nicht nur auf sein sagenhaftes Gedächtnis stützte. ${ }^{10}$ (In der Sekundärliteratur war László Juhász der erste, der die Meinung vertreten hat, daß Janus in Italien kommentierte Bücher benutzt hatte und vor allem das Fehlen solcher Literatur seine Klagen in Ungarn verursachte. $)^{11}$ Guarinos Buch De modo et ordine docendi ac studendi (Verona, 1459) beweist zweifelssfrei, daß im von Guarino vorgeschriebenen Studiengang die lexikographischen Kentnisse eine wichtige Rolle spielten. Die wichtigsten und anerkanntesten Autoren waren unter anderen Gellius, Plinius der Ältere, Macrob und Augustin. ${ }^{12}$ Aber aufgrund der aus diesem Brief gewonnenen Informationen können wir diesen Kreis erweitern.

Der erste Autor wäre Nonius Marcellus. Das Wort ebriositas ,Trunksucht' aus Z. 35 des Briefes fehlt in der von Janus genannten Stelle des Cicero. Das Wort ist in allen Handschriften der Tusculanae Disputationes ausgefallen, und die moderne Textausgaben können das Wort nur aus der indirekten Tradition in den Cicero-Text einfügen. ${ }^{13}$ Die ungewöhnliche Form wurde bloß wegen ihrer Seltenheit und Kuriosität von Nonius Marcellus bewahrt. ${ }^{14}$ Man könnte vermuten, daß Janus Pannonius vielleicht eine seitdem verloren gegangene Handschrift des Cicero-Werkes benutzt hatte, welche noch die vollständigere „Nonius-Lesung“ beinhaltete. Die weiteren Ähnlichkeiten zwischen den Beispielen von Janus und Nonius sind aber entscheidend. Das auf gleiche Weise gebildete und deshalb interessante mulierositas (Z. 34) des Cicero taucht ebenfalls an einer prominenten Stelle des Nonius auf - diesmal hat das Zitat bei Nonius seine handschriftliche Parallele in Cicero. ${ }^{15}$ So hat Janus ohne Zweifel im ersten Fall - obwohl er es unerwähnt läßt - De compendiosa doctrina des Nonius Marcellus benutzt.

Wir können das mit einer gewissen Sicherheit bestätigen, weil die beiden Artikel bei Nonius, wo die erwähnten Wörter angeführt werden, vor den Cicero-Zitaten die Stellen genau angeben: Cicero discrevit Tusculanarum lib. $I V{ }^{16}$ und Cicero Tusculanarum lib. $I V .{ }^{17}$ Aufgrund dieser Referenzen konnte Janus Pannonius einfach die Wörter bei Cicero aussuchen. Wenn unsere Rekonstruktion zutrifft, ist Janus auf diese Weise weiteren Beispielen auf die Spur gekommen. So hat er, vermute ich, das Wort vitiositas (Z. 34) bei Cicero gefunden, welches bei Nonius nicht zu finden ist.

\footnotetext{
${ }^{10}$ HuszTi József, Janus Pannonius (Pécs, 1931) 26 (Battista Guarino hat diese Fähigkeit des Janus bewundert).

${ }^{11}$ JUHÁSZ László, op.cit. 17 und 19.

${ }^{12}$ HuSZTI op.cit. 20.

${ }^{13}$ M. POHLENZ (ed.), M. T. Cicero, Tusculanae Disputationes (Lipsiae, 1918) IV.2 (374).

${ }^{14}$ Non.De Comp.Doctr. 444.M. 1-6. W. M. LINDSAY (ed.) (Lipsiae, 1903) 712. Nonius spielt eine ähnliche und wichtige Rolle in der Textüberlieferung von Sallust cf.: P. T. KEYSER, „Nonius Marcellus' Quotations of Sallust“ WS 109 (1996) 181-226. Zwei Zitate füllen glücklicherweise gerade zwei Lakunen in der direkten Tradition ein (217).

${ }^{15}$ Non.De Comp.Doctr. 142.M. 19-22 (LINDSAY, 207).

${ }^{16}$ Non.De Comp.Doctr. 444.M. 2.

${ }^{17}$ Non.De Comp.Doctr. 142.M. 20.
} 
Tusculanae Disputationes und De Officiis von Cicero spielten, wie wir wissen, eine wichtige Rolle im Studiengang in Ferrara. ${ }^{18}$

Das Stichwort ebriositas befindet sich im fünften Buch des Nonius, dessen Titel lautet: De differentia similium significationum. Es wäre kaum überraschend, wenn Janus dieses Kapitel des Nonius durchgelesen hätte. Janus Pannonius suchte nämlich Beispiele, um die von Nigidius Figulus befürwortete Wortbildungstheorie gegen Gellius zu unterstützen. Das zweite Buch konnte ihm ebenfalls vielversprechend erscheinen, dessen Titel lautet: De (in)honestis et nove veterum dictis per litteras. Dieser Teil der De compendiosa doctrina wurde von dem Autor oder viel wahrscheinlicher von der Nachwelt in alphabetische Ordnung (per litteras) gestellt. Das Stichwort mulierositas zu finden, ist leicht, um so mehr, als Gellius selbst seinen Leser auf das Wort unter den Adjektiven aufmerksam macht, die er für repräsentativ für die Wortbildungs- und Bedeutungsregel des Nigidius hält. ${ }^{19}$

Das Werk des Nonius wurde im Mittelalter weitgehend benutzt. Die Kompilatoren der mittelalterlichen Wörterbücher und Glossare haben die antiken Handbücher meistens dramatisch abgekürzt, epitomiert und aus praktischen Gründen in alphabetische Reihenfolge gebracht. ${ }^{20}$ Ein typisches Beispiel für solche Kompendien ist $\mathrm{Li}$ ber Glossarum, wo auch Nonius-Glossen eingefügt wurden, obwohl es unklar ist, ob es mittels direkter Benutzung des antiken Autors oder indirekt geschah. ${ }^{21}$ Es gibt auch eine selbständige Nonius-Glossensamlung im Codex Leidensis $67 \mathrm{~F}^{22}$ Unter den Stichwörtern dieser Sammlung können wir die Eintragung , mulierositas - mulierum nimia cupiditas $^{6}$ finden. ${ }^{23}$ Hier sind aber die Fundstellen der Zitate überhaupt nicht angeführt, so hat Janus nicht ein solches Glossar benutzt, sondern eine vollständige Nonius-Handschrift, weil er Cicero und sein Werk mit Namen und Titel erwähnt.

Wir können aber die gerade erwähnte Annahme, daß Janus das Werk des Nonius wie ein Handbuch benutzt hat, auch mit indirekten Argumenten bestätigen. In der eindrucksvollen Korrespondenz der Humanisten am Anfang des 15. Jahrhunderts kann man den lebhaften Verkehr der neuentdeckten oder wiedergefundenen Handschriften verfolgen, unter anderen auch Nonius Marcellus selbst. Bartolomeo Capria hat in Pavia in der Visconti-Bibliothek eine Nonius-Handschrift entdeckt, welche er zwischen 1407 und 1409 an Leonardo Bruni Aretino geschickt hat. ${ }^{24}$ Ein anderer Entdecker ist aber viel bedeutsamer für die Janus-Übersetzung. In Paris hat Poggio Bracciolini während seiner Reisen in Europa das Werk des Nonius gefunden. Darüber schreibt er in einem Brief, in dem er seinen Freund, Nicolao, sein Exemplar zurückzuschicken bittet: Hoc ego te rogo permaxime, mittas etiam libellum Nonii Mar-

\footnotetext{
${ }^{18}$ Nach HUSZTI op.cit. 21. Es scheint so zu sein, daß De Natura Deorum (soweit es sich im Buch von Huszti nachvollziehen läßt) nicht so nachdrücklich vorgeschrieben wurde, cf.: unten S. 205.

${ }^{19}$ Gell.Noct.Att. IV.9.12.

${ }^{20}$ Cf.: G. GOETZ (ed.) Corpus Glossariorum Latinorum (=CGL) (Lipsiae, 1884) Vol. I (Reprint Amsterdam, 1965) 91.

${ }^{21}$ CGL I. 104-117, cf.: G. GoETZ, Berl.Philol.Woch. IX (1889) 1331-2.

${ }^{22}$ CGL V. 637.

${ }^{23}$ CGL V. 644.

${ }^{24}$ Phyllis Walter Goodhart GoRDAN, Two Renaissance Book Hunters (New York, London, 1974) 233.
} 
celli, quem ad te misi una cum aliis rebus ex Parisio scriptum litteris antiquis, quod erit mihi maiorem in modum gratum ... $1425 .^{25}$

Sein Freund war sehr an Nonius interessiert: Ut obsequor voluntati tuae mitto ad te per Candidum nostrum antiquum volumen illud Orationum Tullii, et item Nonium Marcellum, quos tantopere postulas, ut videatur totum studium tuum et opera ex illis pendere. At cum illos receperis, scio te repositurum ubi multis mensibus, ne dicam annis, illos non videas, et me prives lectione (1429). ${ }^{26}$

Wahrscheinlich hat Nicolao Niccoli selbst eine Abschrift gemacht und weitergeschickt: ... tu vero illum Silium Italicum, Nonium Marcellum, Orationes Ciceronis uno spiritu vis conscribere; quia omnes dicis, nullum absolves. ${ }^{27}$

Nach dem Tod von Niccoli 1437 wurde seine Handschriftensammlung in die von Cosimo de Medici begründete Bibliotheca Marciana übertragen und ebenso wie alle anderen Bücher der ersten öffentlichen Bibliothek in Florenz dem Publikum zugänglich gemacht. In einem ähnlichen Zusammenhang hat Francisco Barbaro an Nicolao Niccoli geschrieben, daß er gerade mit dem Abschreiben seines Nonius angefangen habe und es gleich zurückschicken werde (Venice, 1415). Thommaso Fergaso hat vor 1414 Francisco Barbaro um einen Nonius Marcellus und die De Lingua Latina des Varro gebeten. ${ }^{28}$

Obwohl die Informationen aus den verschiedenen, eben angeführten Briefen keineswegs in eine logische oder chronologische Reihenfolge gebracht werden können, ist es klar, daß sogar mehrere Nonius-Handschriften in den Händen der italienischen Humanisten verfügbar waren. Das Exemplar des Poggio mag das beste und vollständigste gewesen sein. Janus konnte damit leicht Bekanntschaft machen, weil es sehr wahrscheinlich zu sein scheint, daß Poggio unter anderen auch einen Nonius an seinen anderen Freund, Guarino, den Meister des Janus, geschickt hat. Über solche Beziehungen zwischen den zwei berühmten Humanisten gibt es verschiedene Beweise, wie etwa den Brief Poggios an Guarino, in welchem er über seine andere große Entdeckung, Quintilian, berichtet. ${ }^{29}$ Über ihre Freundschaft hat uns auch Janus berichtet, gar nicht zu reden von seinem Besuch 1458 - obwohl ein Jahr nach der Plutarch-Übersetzung - bei Poggio in Florenz. ${ }^{30}$ Während dieser Reise hat Janus mehrere Bücher von Vespasiano gekauft. ${ }^{31}$

Das im Vordergrund des wissenschaftlichen Interesses stehende Nonius-Werk konnte man gewiß vor 1457 auch in Ferrara finden. Im am 19. Januar 1436 datierten Katalog der Este-Bibliothek steht Nonius Marcellus unter Nummer 276. Nach der Meinung von Sabbadini war dies gerade die Folge von Guarinos Bemühungen. ${ }^{32}$

\footnotetext{
${ }^{25}$ Riccardo FubINI (ed.) Poggius Bracciolini: Opera Omnia I-IV (Torino, 1969) III. 148-9. Die Datierung ist unsicher.

${ }^{26}$ FUBINI op.cit.III. 294.

${ }^{27}$ FUBINI op.cit. III. 150.

${ }^{28}$ R. SABBADINI, Le scoperte dei codici latini e greci nei secoli XIV e XV (Firenze, 1905) 73.

${ }^{29}$ FUBINI op.cit. III. 25.

${ }^{30} \mathrm{~S}$. TELEKI, S. KovÁSZnAY, Iani Pannonii poemata quas uspiam reperiri potuerunt omnia I-II (Traiecti ad Rhenum, 1784). I. 44-5 (im weiteren: TELEKI-KOVÁsZNAY). Das Lobgedicht über Guarino Z. 786-803.

${ }^{31}$ HuszTI op.cit. $176-8$.

${ }^{32}$ SABBADINI op.cit. 198
} 
Das zweite, nicht erwähnte Referenzbuch für Janus war der eben genannte Quintilian.

Janus Pannonius führt in der Reihe der relevanten antiken Testimonien noch vor den eben analysierten Cicero- oder besser Nonius-Zitaten eine andere Wortbildung des Cicero an (Z. 32): sicut illa Ciceronis beatitudo et beatitas. Das Demonstrativpronomen illa sollte in diesem Kontext - glaube ich - als , das Allbekannte oder Legendäre' interpretiert werden. Unter den verschiedenen Gruppen der Zitate des Janus - ich meine Z. 22-7, Z. 32-3, Z. 33-6 - gibt es in seiner Formulierung markante Unterschiede. Die dritte, aus Nonius geschöpfte Gruppe fügt Janus wie einen Zusatz hinzu und erwartet vom Leser offensichtlich nicht, daß er diese Beispiele kennt. Die erste Gruppe (Z. 22-7) stammt im Gegenteil aus Lektüren, die der gelehrte Leser auswendig kennen sollte. (Hierzu gehören Catull und eine Cicero-Paraphrase.) ${ }^{33}$ Die mittlere Gruppe, in der sich beatitas und beatitudo befinden, hat wegen der speziellen Bedeutung des Pronomens illa eine kaum verkennbare Färbung, als ob dieses Exemplar den wissenschaftlichen Kreisen allbekannte wäre.

Tatsächlich tauchen die kühnen Substantive des Cicero an einer prominenten Stelle des Quintilian auf. Bei Cicero lesen wir: sive beatitas sive beatitudo dicendast (utrumque omnino durum, sed usu mollienda nobis verba sunt) ${ }^{34}$ Bei Quintilian dasselbe: qualia apud Ciceronem beatitas et beatitudo: quae dura quidem sentit esse, verum tamen usu putat posse molliri. ${ }^{35}$ Und die Formulierung des Janus Pannonius in Z. 32 lautet: vel sicut illa Ciceronis beatitudo et beatitas, usu aliquando emollescent.

Es ist fast unmöglich zu entscheiden, welcher antike Autor die Quelle für Janus Pannonius war. Als einziger Anhaltspunkt könnte vielleicht betrachtet werden, daß das von Janus Pannonius verwendete futurum imperfectum (emollescent) der potentiellen Bedeutung bei Quintilian inhaltlich näher als der Aufforderung des Cicero steht.

Viel wichtiger kann das Argument sein, daß Janus Pannonius überall in seinem Brief, wo er es tun kann - abgesehen davon, ob er die Originalfassung oder eine Sammlung gelesen hat -, nicht nur den Namen des Quellenautors, sondern auch den Titel des Werkes angibt. In diesem Fall verschweigt er aber das Werk des Cicero genau so, wie es Quitilian tut. Quintilian spricht nämlich über beatitas in seinem Kapitel über die Wortbildung nur beiläufig.

Es ist sehr wahrscheinlich, daß das dritte Kapitel im achten Buch des Quintilian Janus' Interesse geweckt hatte. Er suchte sicherlich nicht nur vereinzelte Beispiele der inkriminierten Wortbildung, sondern mag im allgemeinen einen Versuch gemacht haben, erreichbaren antiken Wortbildungstheorien nachzugehen. Der Titel des besonderen Kapitels lautet: De ornatu in singulis verbis (antiquis, fictis etc.)

\footnotetext{
${ }^{33}$ Catull war in dieser Zeit in Verona weit bekannt. Die erste Edition war von Guarinos Sohn und Enkel besorgt worden. Janus hat Catull neben Martial, Ovid und Vergil als sein poetisches Vorbild angesehen. Cf.: URBÁN László, „Janus Pannonius Catullus-olvasmányai“ in: JPT 397-405.

${ }^{34}$ Cic.De Nat.Deor. I.34.

${ }^{35}$ Quint.Inst.Or. VIII.2.32.
} 
atque coniunctis. De vitiis et virtutibus huius ornatus $;{ }^{36}$ in ihm katalogisiert Quintilian verschiedene Methoden, welche der Ausschmückung des Textes dienen. In dieser Hinsicht können die gebildeten Wörter, verba ficta, eine bedeutende Rolle spielen. ${ }^{37}$ Unter den Beispielen dieser Verbesserungsmöglichkeit steht das erwähnte Cicero-Zitat.

Meiner Meinung nach hat Janus vor allem die Unterkapitel 30 bis 37 benutzt. Hier konnte er ebenfalls eine starke Ermutigung des antiken Stilisten Quintilian finden: Man solle vor gemäßigten sprachlichen Neuerungen nicht zurückschrecken. ${ }^{38}$ Der Neuerer solle, um seine Neuerung vom Publikum akzeptieren zu lassen, dem potentiellen Widerwillen entgegenkommen. Auch der Interpret müsse mit gewissen Wendungen seine neuen Wörter einführen oder sogar umbauen: sed si quid periculosius finxisse videbimur, quibusdam remediis praemuniendum est: „ut ita dicam, si licet dicere, quodam modo, permittite mihi sic uti" quod idem etiam in his, quae licentius translata erunt, proderit, quae non tuto dici possunt. In quo non falli iudicium nostrum sollicitudine ipsa manifestum erit. ${ }^{39}$

Die Spuren dieser Theorie sind vielleicht schon hinter der gesuchten Weigerung des Janus Pannonius gegenüber der Autorität des Gellius erkennbar (Z. 16-17). Derselbe stilistische Eifer ist in seinen künstlerischen Gegenwendungen in Z. 30 offensichtlich: At durum quiddam est negotiositas! usw. Zusammenfassend: Sogar die Formulierung, der Stil des Janus-Briefes scheint eine bewußte Reflektion des Quintilian-Textes zu sein. ${ }^{40}$

Quintilian gibt vielleicht ebenfalls die Antwort auf die Frage, warum Janus Pannonius in seiner Übersetzung auch das Wort curiositas verwendet hat. Im genannten Kapitel des Quintilian konnte er das Adjektiv curiosus in der speziellen Bedeutung lesen, wie er es in der Plutarchischen Schrift verstanden haben wollte: ${ }^{41}$ est etiam quae $\pi \varepsilon \rho \imath \varepsilon \gamma i ́ \alpha$ vocatur, supervacua, ut sic dixerim, operositas, ut a diligenti curiosus et religione superstitio distat. ${ }^{42}$

${ }^{36}$ Es gibt tatsächlich inhaltlich orientierende Indizes in den Quintilian-Handschriften des betrachteten Zeitraums. Cf.: L. RADERMACHER (ed.), V. BuChHEIT (corr.) Quintilianus: Institutio Oratoria (Lipsiae, 1959) X.

${ }_{38}^{37}$ Quint.Inst.Or. VIII.3.24-40.

${ }^{38}$ quae cur tanto opere aspernemur nihil video, nisi quod iniqui iudices adversus nos sumus ideoque paupertate sermonis laboramus. quaedam tamen perdurant. Nam et quae vetera nunc sunt, fuerunt olim nova ... (33-4). Noch nachdrücklicher: ut iam nobis ponere alia, quam illi rudes homines primique fecerunt, fas non sit, at derivare, flectere, coniungere, quod natis postea concessum est, quando desiit licere? (36).

${ }^{39}$ Quint.Inst.Or. VIII.3.37.

${ }^{40}$ Quintilian schreibt auch an anderen Stellen über die Schwierigkeiten der Wortbildung: nova non sine quodam periculo fingimus. Nam si percepta sunt, modicam laudem adferunt orationi, repudiata etiam in iocos exeunt. Audendum tamen: namque, ut Cicero ait, etiam quae primo dura visa sunt, usu molliuntur. Inst.Or. I.5.71-2. Wie es aus diesen Worten klar hervorgeht, war Ciceros Leitgedanke schon für Quintilian etwas Legendäres, oder besser, er hat daraus eine Maxime gemacht. Die Wendung ,primo dura visa sunt‘ bringt uns wieder ein wenig näher zu Janus', aliquando emollescent'.

${ }^{41}$ Quint.Inst.Or. VIII.3.55.

${ }^{42}$ Das Substantiv $\pi \varepsilon \rho 1 \varepsilon \rho \gamma i ́ \alpha$ taucht in Plutarchs Werk viermal auf, Janus aber - obwohl er damit einen entscheidenden Beweis für unsere Annahmen liefern könnte - folgt nicht Quintilian (,operositas ${ }^{\natural}$ ), sondern er benutzt manchmal ,negotiositas' (515.F, 522.B), manchmal, curiositas' (519.C, 521.A). Es ist eine andere interessante Erscheinung, daß Janus in weiteren Fällen der zusammengesetzten griechischen 
Woher kannte Janus Pannonius Quintilian so gut kennen? Die Antwort ist einfach, weil die wichtigsten Autoren in Guarinos Lehrplan für den dritten rhetorischen Studiengang die neu gefundenen Quintilian und Cicero waren. ${ }^{43}$ Es gab sogar ein gewisses Quintilian-Fiber unter den Humanisten der ersten Jahrzehnte des 14. Jahrhunderts. Poggio hat eine vollständige Quintilian-Handschrift gefunden, und darüber hat er gleich seinen Freund Guarino, den Meister von Janus, benachrichtigt. ${ }^{44}$

Nach Nonius Marcellus und Quintilian können wir noch Aulus Gellius als eine von Janus benutzte lexikographische Encyclopedia anführen. Es besteht kein Zweifel, daß Janus die Attischen Nächte in seinen Überlegungen als seinen Ausgangspunkt angesehen hatte. Auf welche praktische Weise er aber Gellius benutzte, darüber gibt es noch ein interessantes Indiz. In Z. 15 sagt Janus, wie oben schon zitiert, daß Gellius sehr oft curiosus in der Bedeutung accuratus, sorgfältig' benutzt. Zwei signifikante Stellen - von insgesamt zehn -, wo Gellius das Adjektiv in dieser Bedeutung benutzt, kommen nur in den lemmata, den kurzen inhaltlichen Zusammenfassungen vor (XIII.1 ,curiosior ${ }^{6}$ und XVIII.15 ,curiosae $)$ und fehlen im Text der Kapitel. ${ }^{45}$ An weiteren 14 Stellen kommen Bildungen aus dem Adjektiv ,curiosus' vor, von welchen zwei ebenfalls nur im ,lemma (VI.7 und I.25) und ein drittes im , lemma und im Text (I.4, I.4.1) auftaucht. Von den acht Stellen, wo ,curiosus' (in der Bedeutung , anxius ${ }^{6}$ ) im Text integriert vorkommt, ist es dreimal mitten in linguistische Probleme eingefügt, welche für die Wortbildungsfragen relevant sind. ${ }^{46}$ Höchstwahrscheinlich hatte Janus Pannonius also zuerst immer wieder die ,lemmata' durchgelesen, ungefähr so, wie es wir heute mit einem solchen antiquarischen Werk tun würden, wenn es sich um lexikographische Probleme handelt. Auf diese Weise mag

Begriffe nach Erwiderung des Griechischen mit Vorliebe umschrieb. Zwei Beispiele dafür: $\dot{\varepsilon} \chi \varepsilon \mu v \theta i ́ a v$

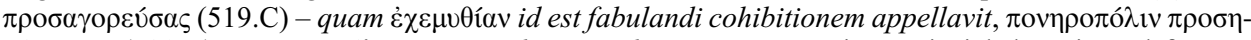

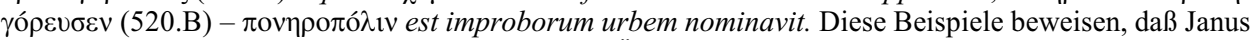
zwar wegen der Unvollkommenheit der lateinischen Übersetzung nicht zufrieden war, aber er keine Neuerungen schaffen und die klassischen Autoren übertreffen wollte. Im Falle der , negotiositas ' macht er nichts anderes, als daß er eine verworfene, aber trotzdem einigermaßen existierende Lösung befürwortet.

${ }^{43}$ Cf.: HuszTI op.cit. 21.

${ }^{44}$ Quo uno solo (sc. Quintiliano), etiam si Cicero Romanae parens eloquentiae deesset, perfectam consequeremur scientiam recte dicendi. Is vero apud nos antea, Italos dico, ita laceratus erat, ita circumcisus, culpa, ut opinor, temporum, ut nulla forma, nullus habitus hominis in eo recognosceretur. Tute hominem vidisti hactenus ... lacerum crudeliter ora, / Ora manusque ambas populataque tempora raptis / Auribus, et truncas inhonesto vulnere nares. ... Nam mehercule nisi nos auxilium tulissemus, necesse erat illum propediem interiturum ... Est autem monasterium Sancti Galli prope urbem hanc mil. pas. XX. ... Ibi inter confertissimam librorum copiam quos longum esset recensere, Quintilianum comperimus adhuc salvum et incolumem, plenum tamen situ, et pulvere squalentem ... Haec mea manu transcripsi, et quidem velociter, ut ea mitterem ad Leonardum Aretinum, et Nicolaum Florentinum; ... Habes mi suavissime Guarine, quod ab homine tibi deditissimo ad praesens tribui potest. Vellem et potuisse librum transmittere, sed Leonardo nostro satisfaciendum fuit. Verum scis quo sit in loco, ut si eum voles habere, (puto autem te quam primum velle) facile id consequi valeas. Vale, et me, quando mutuum fit, ama. Constantiae XVIII. Kalendas Januarias Anno Christi 1417. Cf.: FUBINI op.cit. III. 25-29.

${ }^{45}$ Nach den Angaben der TLL kommt bei Gellius 2- (8-) mal curiosus in der Bedeutung, anxius, attentus, nimis diligens' vor. Die zwei hervorgehobenen Stellen: XIII.1 (curiosior) und XVIII.15 (curiosae) finden sich nur in den vorangehenden des Kapitels vor sowie in deren inhaltsverzeichnismäßiger Aufzählung, ganz am Anfang des Werkes, sie fehlen im Text der Kapitel vollkommen.

${ }^{46}$ Die acht Stellen sind die folgenden: XVI.12.1; II.17.1; XIX.13.1; III.3.1; VII.14.3; XVI.12.1; XVII.2.11; XVIII.9.5. 
Janus die thematisch wichtigsten zwei kurzen Abhandlungen des Gellius gefunden haben, im ,lemma' zu XI.16: Quod Graecorum verborum quorundam difficillima est

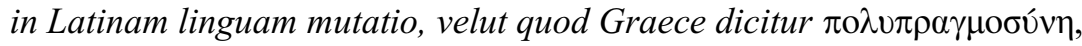
und im ,lemma' zu IV.9: Quid significet proprie ,,religiosus "; et in quae deverticula significatio istius vocabuli flexa sit; et verba Nigidii Figuli ex commentariis eius super ea sumpta.

Hier sei ebenfalls an die Stelle Quintilians erinnert, wo er den Unterschied zwischen den Bedeutungen von, diligens ${ }^{6}$ und ,curiosus ${ }^{6}$ untersucht und als eine Parallele ,religio' mit ,superstitio ' kontrastiert. $^{47} \mathrm{Im}$ Lemma fällt gleich auf, daß Gellius gerade über Quintilians erklärendes Beispiel etwas zu sagen hat und deswegen ist es vielleicht kein Zufall, daß auch Janus in seinem Brief (Z. 21) das Adjektiv ,religiosus` erwähnt.

Die intensive Benutzung des Gellius als ein mit Index versehenes Referenzbuch würde ebenfalls erklären, warum Janus unter anderem gerade das Adjektiv , vitiosus' aus Cicero noch anführt (Z. 34). Es steht ebenfalls bei Gellius, im zwölften Kapitel des dritten Buches, wozu das Lemma lautet: Largum atque avidum bibendi a P.Nigidio, doctissimo viro, nova et prope absurda vocabuli figura bibosum dictum.

Eine weitere, nicht beantwortete Frage ist, woher Janus wußte (Z. 22-24), daß Cicero das Adjektiv ,curiosus' im ersten Buch von De Officiis in diesem Sinne, also für $\pi \circ \lambda v \pi \rho \alpha ́ \gamma \mu \omega v, \pi \mathrm{o} \lambda v \pi \rho \alpha \gamma \mu \circ \sigma u ́ v \eta$ benutzte.

Die Übersetzung $\pi \mathrm{o} \lambda \nu \pi \rho a ́ \gamma \mu \omega v$, curiosus ${ }^{6}$ konnte einfach aus einem Vocabularium stammen. Im Mittelalter wurden neben den Glossarien, die meistens einsprachige Wörterbücher waren, Hermeneumata als zweisprachige zusammengefügte Wörterlisten benutzt, die auch unter den Humanisten sehr beliebt waren. In diesem Fall könnten nur zwei bekannte Hermeneumata in Frage kommen, weil nur in Hermeneuma Monacensia und Einsidlensia das Paar $\pi \mathrm{o} \lambda \nu \pi \rho \alpha ́ \gamma \mu \omega v$ - ,curiosus ${ }^{6}$ vorkommt. ${ }^{48}$ Die zwei Hermeneumata sind ihrem Wesen nach sehr ähnlich, abgesehen davon, daß die Struktur von Einsidlensia vereinfacht ist. Diese Wörterbücher waren für Anfänger in Griechisch zusammengestellt, man konnte in zwei Kolumnen nebeneinander einen zusammenhängenden Text in Griechisch und Lateinisch Wort für Wort verfolgen. Die zweite Hälfte der Hermeneumata ist aber thematisch geordnet. Der Abschnitt De moribus humanis (14-e in Mon. und 28-e in Ein.) enthält das Adjektiv $\pi \mathrm{o} \lambda \nu \pi \rho a ́ \gamma \mu \omega v$, curiosus $^{\text {، }}{ }^{49}$ Welche von diesen Hermeneumata in der Hand von Janus gewesen sein könnte, ist nicht zu entscheiden.

Das bekannte Vocabularium des Janus, won dem István Kapitánffy überzeugend nachgewiesen hat, daß es - trotz der inzwischen verlorengegangenen einleitenden , notitia ${ }^{\text { }}$ - nicht von Janus geschrieben, sondern nur benutzt wurde, ist nur eine humanistische Kopie des von Goetz beschriebenen Kodex Harleianus aus dem 7. Jahrhundert. ${ }^{50}$ Die griechischen Ergänzungen und literarischen Zitate am Rand des

\footnotetext{
${ }^{47}$ Cf.: Anm. 41.

${ }^{48}$ CGL III. 117 und III. 220.

${ }^{49}$ CGL III. 179.52 und III. 251.76.

${ }^{50}$ ÖNB Suppl. Gr. 45. Cf.: Anm. 2.
} 
Vocabularium führen uns - nach der Meinung von Kapitánffy - zu Guarino. Von ihm hat es Janus nach dem Ende seiner Studien vermutlich gekauft und nach Ungarn mitgebracht.

Die Einträge dieses Wörterbuches geben keine entscheidende Antwort, ob die lateinischen Begriffe in der Plutarch-Übersetzung von Janus von hier stammen. Neben $\pi \circ \lambda \cup \pi \rho \alpha \gamma \mu$ ov $\tilde{\omega}-\varepsilon \tilde{\imath} \varsigma$ stehen in der ursprünglichen Kolumne als lateinische Äquivalente die Verben ,sciscitor ${ }^{6}$ und ,percontor ${ }^{6}$. Als Ergänzung zu diesen hat man nach der Beendigung der Wörterliste wahrscheinlich in drei verschiedenen Phasen, daß heißt mit drei verschiedenen Händen, die folgenden Varianten eingefügt: 1. auf den durch das Verso angebotenen breiten linken Rand, in rebus nihil pertinentibus implicor ${ }^{6}, 2$. zwischen den beiden Kolumnen ,negotior ${ }^{6}$ und 3. zum schließlich neben den ursprünglich angegebenen zwei lateinischen Verben das schwer lesbare ,discurro' oder ,discurso'. Das Adjektiv $\pi$ o $\lambda \nu \pi \rho \alpha ́ \gamma \mu \omega v$ hat nur ein

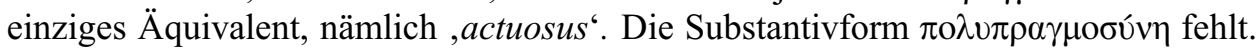
Neben dem verwandten $\pi \varepsilon \rho ı \varepsilon \rho \gamma a ́ \zeta o \mu \alpha \iota$ steht ,satago'. Als weitere Ergänzungen von anderer Hand sind auf dem durch das Recto angebotenen breiten Rande zu lesen: ,sciscitor, curiose ago, elaboro, orno'. Neben dem Adjektiv $\pi \varepsilon \rho i ́ \varepsilon \rho \gamma o \varsigma$ stand in der ersten Version nur ,curiosus', und erst später wurden ,supervacaneus' und ,superabundans' hinzugefügt.

Wenngleich die Äquivalenz $\pi 0 \lambda v \pi \rho \alpha ́ \gamma \mu \omega v$,curiosus` eine aus Wörterbüchern stammende Selbstverständlichkeit für Janus war, ist es eine noch offene Frage, woher Janus die Kenntnis hatte (Z. 22), daß Cicero an dieser Stelle von De Officiis das Adjektiv ,curiosus' in dieser Bedeutung benutzt hat, oder genauer gesagt, eine gewisse Stelle der Politeia Platons vor sich hatte. ${ }^{51}$ Platon hatte nämlich im vierten Buch der Politeia mehrmals die Substantiv- und Adjektivformen dieses Begriffes angewandt. ${ }^{52}$ Diese Frage kann nur so beantwortet werden, daß er es gewußt hat. Janus Pannonius hatte das griechische Original gekannt, weil es gerade zu dieser Zeit neu entdeckt und ins Lateinische übersetzt wurde. In Guarinos Curriculum Studii finden wir ebenso die Werke von Cicero wie auch einige von Platon. ${ }^{53}$ Die Stelle, über die Cicero

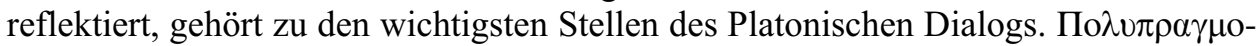
$\sigma o v \eta \eta$ repräsentiert in der Politeia von Platon keinen Nebenbegriff, sondern ist das

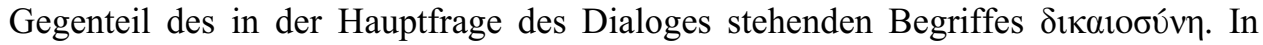
Platons idealem Staat, wo Gerechtigkeit herrscht, sollte jedermann seine Aufgabe tun und ein unberufenes Einmischen in die Angelegenheiten und Gerechtigkeit $-\pi \mathrm{o} \lambda v$ $\pi \rho \alpha \gamma \mu \sigma \sigma \delta ́ v \eta$ - vermeiden.

${ }^{51}$ Cf.: App. I.22-3; Cic.De Off. I.34: Privatum autem oportet aequo et pari cum civibus iure vivere neque summissum et abiectum neque se ecferentem, tum in re publica ea velle, quae tranquilla et honesta sint, talem enim solemus et sentire bonum civem et dicere. Peregrini autem atque incolae officium est nihil praeter suum negotium agere, nihil de alio inquirere minimeque esse in aliena re publica curiosum.

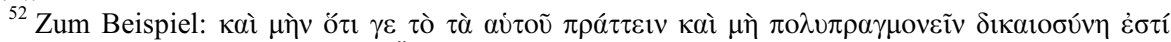
Plat.Resp. IV.433.a. In der lateinischen Übersetzung von Ficino (nach Hirschig op.cit.): „Atque sua agere neque aliena curare iustitiam esse item cum ex aliis multis audivimus, tum ipsi saepe diximus." Für $\pi$ oגv-

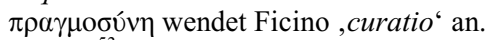

${ }^{53}$ HUSZTI op.cit. 21. 
Als Janus Pannonius über die Übersetzung der $\pi 0 \lambda v \pi \rho \alpha \gamma \mu$ erinnerte er sich wahrscheinlich zuerst an Platon und das Adjektiv, curiosus ${ }^{6}$ bei $\mathrm{Ci}$ cero. Aus dem Adjektiv mußte man aber ein Substantiv bilden. So kam er zu dem von Gellius aufgeworfenen und gleich verworfenen Vorschlag: ,negotiositas", das in Lateinischen ein Hapax ist. Um dieses ,negotiositas' und vor allem die Möglichkeit dieser sprachlichen Neuerung zu retten, hat Janus ein Arsenal von antiken Beispielen angeführt. Er hat die kürzlich wiederentdeckte grammatische, antiquarische und lexikographische Literatur als benutzbare Handbücher weitgehend konsultiert und seine Argumentation auf sie basiert. Schließlich ist er bei dem Vorschlag von Gellius geblieben, wie das der von ihm angegebene lateinische Titel der Abhandlung Plutarchs zeigt. Auf der anderen Seite hat er das Cicero-Testimonium nicht beiseite gelegt, sondern neben ,negotiositas' ${ }^{6}$ auch ,curiosus ${ }^{6}$ und die andere ebenfalls von Cicero benutzte Substantivform ,curiositas ${ }^{6}$ angewandt. ${ }^{54}$ Die Form des letzteren entsprach auch der Regel von Nigidius und seiner Forschungen.

Es ist auf der anderen Seite auffallend, daß der Freund und Zeitgenosse von Janus, Marsilio Ficino, denselben Begriff bei Platon später mit ,curatio ${ }^{6}$ übersetzt hatte. Deswegen ist es interessant, daß Janus in einem seiner Briefe seinen Freund vielleicht ironisch mit den folgenden Worten warnt: Equidem te amice moneo, caveas, ne forte curiositas quaedam sit isthaec renovatio antiquorum, potius quam religio. ${ }^{5}$

Obwohl Janus die Einstellung der vollständigen Platon-Übersetzung nicht erlebt hat, können wir mit Recht vermuten, daß er einige Teile davon gesehen hat. Ein glänzender Beweis für ihre Freundschaft und ihr gemeinsames Platon-Interesse bedeutet Ficinos Widmung für Janus 1469 in seinem Commentarium in Platonis Convivium de amore. ${ }^{56}$

Janus Pannonius war in Italien, wie es wir seinem Brief entnehmen können, in einer inspirierenden geistigen Umwelt mit literarischen Neuigkeiten und von einem verständnisvollen Publikum umgeben. Er konnte sogar in solchen Fällen lexikographische „Handbücher“ konsultieren. Aus anderen Quellen wissen wir, daß die Plutarch-Übersetzungen des Janus nicht isolierte Erscheinungen solcher Bestätigung waren, sondern Marco Aurelio viele Zeitgenossen zum Übersetzen angeregt hat. ${ }^{57}$ Zsámboky (Iohannes Sambucus) hat selbst zwei Übersetzungen der Viten Plutarchs

${ }^{54}$ Cic.ad.Att. 2.12.2. Nach Guarinos Syllabus könnten Ciceros Briefe den Studenten zugänglich gewesen sein (cf.: HUSZTI op.cit. 21). Ich danke Zoltán Farkas dafür, daß er mir die folgenden Resultate seiner Komparativforschungen im lateinischen Wortschatz der Übersetzungen des Janus mitgeteilt hat:

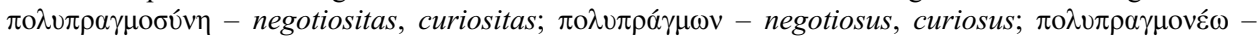
curiosus sum, curiosior inquirere soleo, inquiro 3 , satago 1 , considero 1 , rimor 1 , contemplor 1 , intendo

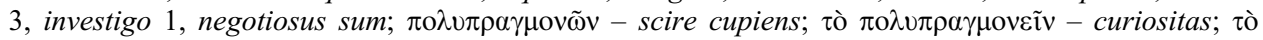
$\pi \mathrm{o} \lambda \nu \pi \rho \tilde{\alpha} \gamma \mu \mathrm{ov}$ - curiositas, negotiositas.

${ }_{55}$ ÁBEL Jenő (ed.), HEgEDŰs István (aux.), Analecta nova ad Historiam renascentium in Hungaria Litterarum spectantia (Budapest, 1903) 278.

${ }^{56}$ ÖNB Cod.Lat. 2472. Cf.: CSAPODI op.cit. 193.

${ }^{57}$ HuszTI op.cit. 156-8. 
von Janus gesehen, in denen Guarinos Anmerkungen erkennbar waren. ${ }^{58}$ Gewiß könnten die den Freunden gewidmeten Einführungen in den Briefen des Janus als literarische Wendungen und Formalitäten angesehen werden, wenn er zum Beispiel die Initiative seiner Übersetzungen Marco Aurelio zuschreibt. Andererseits ist es klar, daß es in seinem im Dezember 1456 geschriebenen Brief keine bloße Höflichkeit ist, wenn er von konkretem Diktieren erzählt. Marco Aurelio hat nämlich die erste Übersetzung von Janus selbst abgeschrieben und vermutlich gleich korrigiert. Diese Beziehungen zwischen den beiden erklären die umständliche Abhandlung von Janus, in welcher er seine Wahl - ,negotiositas " - hauptsächlich seinem Freund erklären und sie verteidigen will.

Die in Ungarn geschriebenen Briefe klagen gerade über den Mangel dieser erwähnten zwei determinierenden Umstände: Er hat kein solches Publikum, das die Probleme verstehen und besprechen konnte, und es gibt keine Bücher. ${ }^{59}$ Der Gegensatz zwischen Italien und Ungarn ist für Janus haupsächlich in diesen zwei Punkten signifikant. Über eine gewisse Gruppe der vermißten ,codices ' können wir aus dem analysierten Brief einen klaren Eindruck bekommen. Janus Pannonius hat auch in Ungarn Plutarch übersetzt, die Apophtegmata sind König Matthias Corvinus gewidmet und wurden wahrscheinlich nicht aus rein wissenschaftlichem Interesse oder aufgrund freundlicher Ermutigung, sondern aus politischen Gedanken verfertigt.

\section{AUSBLICK}

Es ist unwahrscheinlich, daß Janus in seinem Brief an Ficino das Substantiv ,curiositas ${ }^{6}$ deswegen gewählt hat, weil er ihn darauf aufmerksam machen wollte. Auch wenn er es so gewollt hätte, hat er Beifall gefunden. Dasselbe Schicksal hat der Titel ,de negotiositate " teilen müssen, weil in der lateinischen Titelliste der Werke Plutarchs heute De curiositate zu lesen ist. Wie ist es dazu gekommen?

Im 1572 erschienenen und später von Didot neu gedruckten Thesaurus Linguae

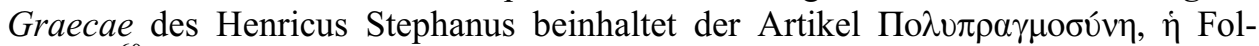
gendes: ${ }^{60}$ Solicitudo de multis negotiis, et quidem nihil ad nos pertinentibus: unde

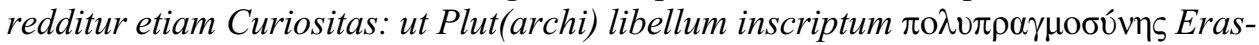
mus vertit De curiositate. At Gellii interpretatio [Negotiositas, N.A. 11, 16] mihi non

${ }^{58}$ JUHÁSZ op.cit. 16. Diese Übersetzungen lagen - wie Juhász meint - kaum über dem Niveau der obligatorischen Schulübungen. Abgesehen von dieser Möglichkeit konnte Janus im Falle der De Negotiositate kaum seinen Meister um Rat bitten, weil er den Winter 1456/7 fern von Ferrara und Padua in der Montagna verbracht hat (cf.: HUSZTI op.cit. 151).

${ }^{59}$ An Marco Aurelio (TELEKI-KovÁSZnAY op.cit. 70-1) 9-10 und 9; An Galeotto Martio (TELEKI-KOVÁSZNAY op.cit. 74-7)10-11 und 9-10.

${ }^{60}$ 1413.col. Im zweiten Teil ist die andere selten verwendete positive Bedeutung des Begriffes erklärt: Inquisitio, Studium res gestas cognoscendi, ap. Polyb. 2,43,9, sigillatim quum quis ipse loca adit quibus res sunt gestae et percunctatur ex hominibus qui rebus interfuerunt, 12,27,6. Die erste zitierte Stelle des Polybius war schon oben angeführt. Hier steht es wegen eines einfachen Druckfehlers, weil der Autor hier über die zum Nachteil der Achaier übertriebene Neugierde des Antigonos Gonatas spricht. Die zweite gehört zu der historiographischen Konfession des Polybius, die besagt, daß der Geschichtschreiber eine gewisse positive Neugierde und Forschungsbestreben haben muß. 
placet: quam vide apud Bud(aeum) p. 306 [Aristoph(anes) Ach(arnanes) 833; Lysias

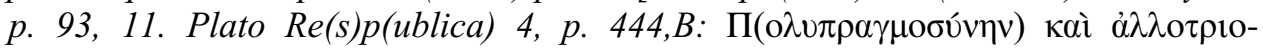

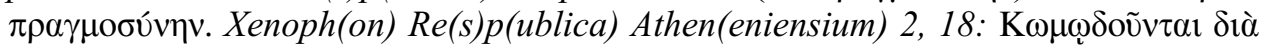
$\pi(\mathrm{o} \lambda \cup \pi \rho \alpha \gamma \mu о \sigma u ́ v \eta v)$. Polyb(ius) 2, 43, 9. Plut(archus) Comp(aratio) Lyc(urgi) et Nu-

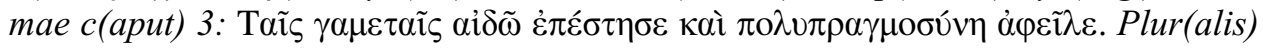

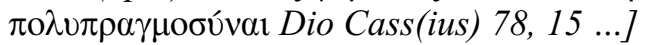

Stephanus beruft sich also auf der einen Seite auf eine ungenannte Quelle (auf die auch in den Werken von Budé zu findenden Äquivalente), auf der anderen Seite folgt er seinem eigenen Urteil (das Wort ,sollicitudo') und umschreibt den Begriff. Stephanus erkannte im wesentlichen die Autorität des Erasmus (,curiositas ${ }^{6}$ ) an und wies die Übersetzung des Gellius aufgrund von Budés Meinung zurück. Das referierte Werk von Budé ist wahrscheinlich das griechisch-lateinische Lexikon, das vier Jahre vor dem Thesaurus 1568 in Basel erschien. ${ }^{61}$ Dieses Lexikon hatte nämlich sehr viel Material dem 1556 in Basel gedruckten anderen Monumentalwerk von Budé, den lexikographisch strukturierten Commentarii Linguae Graecae zu verdanken. Die in eckigen Klammern angeführte ,negotiositas'-Referenz bei Stephanus ist aber irreführend, sie ist eine erst spätere mechanische Ergänzung im Text des Thesaurus, weil Budé an den erwähnten Stellen nicht das Wort ,negotiositas ' bespricht, sondern die von Gellius wirklich befürwortete Umschreibung, die auf der anderen

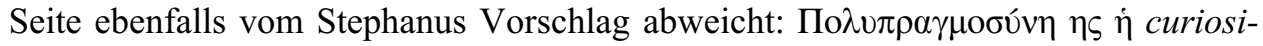
tas, negotiatio, multiplex studium. Gell. vertit multarum rerum aggressionem, earumque omnium actionem, lib. 11. cap. $16 \ldots{ }^{62}$

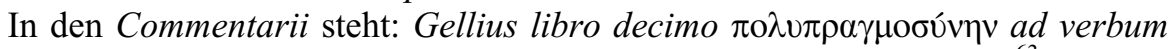
interpretatur multarum rerum aggressionem, earumque omnium actionem. ${ }^{63}$

So hat höchstwahrscheinlich auch Budé ,negotiositas' nicht für adäquat gehalten, deswegen schweigt er darüber und führt die vorgeschlagene Umschreibung nur wegen der Autorität des antiken Autors an, weil Gellius den Begriff, Wort für Wort“ ,ad verbum ' interpretiert hatte. Dieses letztere hat Stephanus' Beifall nicht gefunden, obwohl er den lateinischen Übersetzungen von Budé in seinem Thesaurus meistens genau folgt. ${ }^{64}$

${ }^{61} \Lambda \mathrm{E} \Xi \mathrm{IKON}$ E $\Lambda \Lambda \mathrm{HNOP \Omega MAIKON}$ hoc est Dictionarium Graecolatinum supra omnes editiones postremo nunc hoc anno ex variis et multis praestantioribus linguae Graecae authoribus, commentariis, thesauris et accessionibus, non duntaxat allegationum, sed etiam plurimarum vocum simplicium auctario locupletatum, illustratum et emendatum. In: G. Budeum, L. Tusanum, C. Gesnerum, H. Iunium, R. Constantinum, Io. Hartunqum, Mar. Hopperum Basiliae, 1568 (Im weiteren: Lexikon).

${ }^{62}$ Lexikon s.v.

${ }^{63} 419 . \mathrm{col}$.

${ }^{64}$ Die Bedeutung der Tätigkeit (verbum) nach Stephanus (1412.col.): Multis negotiis me implico, iisque nihil ad me pertinentibus. Plato Rep. 4 [p. 433,A] ... Nach der Meinung der Verfasser des Lexikons (Budé) (cf.: Lexikon s.v.): anxie nimis et curiose aliquid inquiro et indago, satago, anxio nimioque

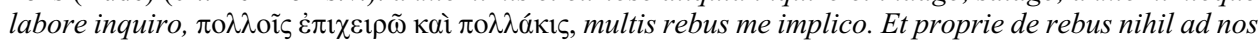
pertinentibus dicitur ... In der eigenen Formulierung von Budé (Commentarii 419.col.): ... est multis sese negotiis implicare, aliena curare: hoc est negotium sibi curiose exhibere, ut sycophantae faciunt. Vitio enim id vertitur ut apud Platonem libro IIII. de Repub. ..., und ein wenig weiter unten übersetzt er ein Partizip in einem Aemilius Apophtegma von Ps.Plutarch folgendermaßen: qui (munia imperatoria) usurpabant nihil ad se pertinentia, hoc est curiose alienis functionibus se implicantes. 
Johannes Forben hat ohne weitere Erklärungen über die Fragen der Übersetzung zwei Plutarch-Übersetzungen des Erasmus 1525 in Basel publiziert, die De cohibenda iracundia und die De curiositate. ${ }^{65}$ Weswegen Erasmus gerade diese (insgesammt elf) Traktate des Plutarch übersetzt hat, ist nicht ganz klar. Er war gewiß ein entschiedener Anhänger der Schriften Plutarchs, deswegen kritisiert er enthusiastisch einige Plutarch-Übersetzungen in seinen Briefen (No. 2422 und 2291). Erasmus konnte in den Jahren 1506-1507 während seiner Reisen in Italien einige Handschriften der Moralien- und Viten-Übersetzungen kennengelernt haben, aber nur spärlich hat er die Namen der Verfasser erwähnt. ${ }^{66}$ Über Janus Pannonius und De negotiositate schweigt er. Im Brief 2291 übt er harte Kritik an den Übersetzungen von Theodoros von Gaza, Georgios von Trapezos und Budé, aber diese Autoren haben $\pi \varepsilon \rho \grave{i} \pi 0-$ $\lambda v \pi \rho \alpha \gamma \mu$ $\sigma 0 ́ v \eta \varsigma$ ins Lateinische nicht übersetzt. ${ }^{67}$ Wir können also De curiositate als eine eigenständige Lösung des Erasmus ansehen. Er hat ,negotium ' und seine Derivata völlig eliminiert. Das Schweigen des Erasmus hat das Schicksal des von Gellius auf- und zugleich verworfenen Vorschlags determiniert. Der Autorität des Erasmus ist nämlich der erste Übersetzer der vollständigen Moralia, Xylander, im Jahre 1570 gefolgt. Die lateinische Übersetzung von Xylander hat Stephanus neben dem griechischen Text in seiner zweisprachigen Edition in den Jahren 1599, 1620 und 1624 gedruckt. ${ }^{68}$ So wurde diese Version und der Titel De curiositate weithin bekannt, und so kann merkwürdigerweise in der Janus-Ausgabe von Teleki und Kovásznay sogar die Übersetzung von Janus wegen der leichteren Identifizierung unter dem Titel $D e$ curiositate stehen.

Das Vocabularium des Janus Pannonius führt als weitere mögliche lateinische Übersetzung für

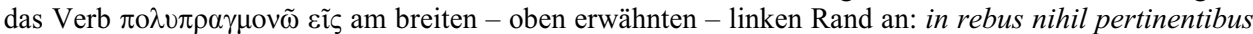
implicor, welches somit meiner Meinung nach den Einfluß der Wörterbücher aus dem 16. Jahrhundert zeigen mag. Die Feststellung von Kapitánffy, daß in diesem Kodex mehrere Hände erkennbar seien, kann in diesem Fall sicher unterstützt werden. Alle drei Einträge am linken und am rechten Rand und zwischen den grieschischen und lateinischen Kolumnen sind klar von der ursprünglichen lateinischen Kolumne zu unterscheiden. Die Hand, die das zwischen den Kolumnen stehende negotiare hinzugefügt hat, repräsentiert eine dritte Version. Wenn wir der Argumentation von Kapitánffy folgen, wonach Janus dieses Vocabularium nicht geschrieben, aber sicherlich benutzt hat, besteht sogar die Möglichkeit, daß diese letzterwähnte Ergänzung Janus selbst zugefügt hat. Er hatte nämlich ein großes Interesse an dem Substantiv negotium und seinen Derivata. Obwohl in den Werken von Budé mehrere Adjektivformen dieses Begriffes vorhanden sind, ist negotiare nicht zu finden.

${ }^{65}$ Cf.: A. J. Koster (ed.), „Ex Plutarcho versa“ in: Opera Omnia Desiderii Erasmi Roterodami, recognita et adnotatione critica instructa notisque illustrata, Ordinis Quarti, Tomus Secundus (Amsterdam, Oxford, 1977) 108.

${ }^{66}$ Cf.: KOSTER op.cit. 107.

${ }^{67}$ Die Plutarch-Übersetzungen von Budé sind: De placitis, De tranquililitate animi, De fortuna Romanorum, De Alexandri fortuna vel virtute. Cf.: David O. MCNEIL, Guillaume Budé and Humanism in the Reign of Francis I (Genf, 1975) 14.

${ }^{68}$ Praef. Mor.I, p.XIII (POHLENZ). 


\section{ANHANG \\ Ante libellum Plutarchi, De curiositate, Latine versum. ${ }^{69}$ Ioannes Pannonius Marco Aurelio S.}

Iterum me acerrimis cohortationibus tuis, Marce Aureli, ad interpretandi officium, quod antea constantissime deprecari consueveram, impulisti. Accipe ergo iterum ex eodem Plutarcho libellum, mores negotiosorum hominum comice depingentem; quem

5 ideo studiosis non inutilem fore arbitror, quod sive hoc morbo laborantes eum legent, poterunt sanari; sive plane valentes, qualis tu es, poterunt oblectari. Ceterum si mandatum meum de eo expectas; ad superioris opusculi, cui et hoc annectendum curabis, praefatiunculam te remitto. Nam in praesentia maius quippiam

10 tecum agere constitui.

Nomen quidem istud, quod pro indice libri ascriptum vides, Aulus Gellius, opinor in Noctibus Atticis, ${ }^{70}$ de hoc ipso tractans ita respuit, ut aliud statim concinnius requirat, postremo desperata eius inventione, quid $\pi \circ \lambda v \pi \rho \alpha \gamma \mu \circ \sigma u ́ v \eta$ Latine significet,

15 longo verborum ambitu describit. Idem, oppido quam frequenter, pro accurato ponit curiosum. Ego tamen, bona venia docti huius et diserti auctoris, eo vitio praeditos partim negotiosos, partim curiosos, vitium ipsum tum negotiositatem tum curiositatem appello. Cum praesertim Nigidius, homo ipsius iudicio, ${ }^{71}$ iuxta

20 Varronem doctissimus, affirmaverit, inclinamentum semper huiuscemodi verborum, ut vinosus, religiosus, signare copiam quandam immodicam rei, super qua diceretur. Cicero etiam potissimum in libro De officiis primo, ${ }^{72}$ cum admonet peregrinum minime esse debere in aliena re publica curiosum; poeta item Catullus, ${ }^{73}$

25 Latinitatis aeque diligens custos, cum ita loquitur:

Quae nec pernumerari curiosi possint, nec mala fascinare linqua manifeste hoc vocabulum, in malum tantum accipiunt. Nos igitur hunc et si praeterea itidem eo utuntur, iure optimo secuti sumus.

30 At durum quiddam est negotiositas! Credo item et curiositas. Sed haec et complura huismodi vel propter necessitudinem facile

${ }^{69}$ In: TeLEKI-KovásznAY II. Bd. 72-4. Die Betonungen und Noten sind von mir. Janus Pannonius hat seiner Übersetzung sicher den Titel De negotiositate gegeben. Das geht klar aus seinem Brief, Z. 11-13, hervor, Gellius spricht nämlich über , negotiositas'. In dieser Form in TELEKI-KOVÁsZNAY 22: Plutarchi Chaeronaei philosophi libellus De negotiositate, Ioanne Pannonio interprete.

${ }^{70}$ Gell.Noct.Att. XI,16.

${ }^{71}$ Gell.Noct.Att. IV,9.

${ }^{72}$ Cic.De Off. I,34.

${ }^{73}$ Cat.VII. 
tolerabuntur, vel sicut illa Ciceronis beatitudo et beatitas, ${ }^{74}$ usu aliquando emollescent. Quamquam idem Cicero in Tusculanis Quaestionibus a mulieroso mulierositatem, a vitioso vitiositatem,

35 ab ebrioso ebriositatem, et multa passim id genus intrepide ac sine ulla nausea configurat. ${ }^{75}$

Verum supra dicta aliquot vocabulorum novitati patrocinantur. Quod si toto in opere dicendi venustatem desiderabis, fac primum cogites; quam aegre procedat omnis traductio; tum quod

40 nec orationes nec historiae tam sunt difficiles translatu, quam scripta philosophorum, in quibus si modo sententiam recte verteris, magna felicitas est cetera non omnino insipida videri. Vale

Patavio, pridie Kal. Mart. M. Quadringentesimo Quinquagesimo septimo.

Eötvös-Loránd-Universität

Institut für Klassische Philologie

H-1364 Budapest, Pf. 107

\footnotetext{
${ }^{74}$ Cic.De Nat.Deor. I.54.

${ }^{75}$ Cic.Tusc.Disp. IV.11 (mulierositas); IV.13,15 (vitiositas); IV.12 (ebriositas).
} 